\title{
Sexual health service adaptations to the coronavirus disease 2019 (COVID-19) pandemic in Australia: a nationwide online survey
}

\author{
Tiffany R. Phillips, ${ }^{1,2}$ Christopher K. Fairley, ${ }^{1,2}$ Basil Donovan, ${ }^{3,4}$ Jason J. Ong, ${ }^{1,2}$ Anna McNulty, ${ }^{4}$ Lewis Marshall, ${ }^{5}$ \\ David J. Templeton, ${ }^{3,6}$ Louise Owen, ${ }^{7}$ Alison Ward, ${ }^{8}$ Manoji Gunathilake, ${ }^{9}$ Darren Russell, ${ }^{10}$ Julian Langton-Lockton, ${ }^{11}$ \\ Christopher Bourne, ${ }^{3,4,12}$ Sarah Martin, ${ }^{13}$ Eric P.F. Chow ${ }^{1,2,14}$
}

\begin{abstract}
$\mathrm{n}$ response to the COVID-19 pandemic,
Australia went into a nationwide

lockdown that began on 22 March 2020

with stage one restrictions. These initial restrictions included closing non-essential businesses as well as limiting the size of social gatherings and implementing 1.5 metre social distancing. ${ }^{1}$ From 29 March 2020 to 8 May 2020, further stage two restrictions were announced across Australia, wherein people were asked to remain inside their homes unless shopping for essential items, exercising, going to essential work or school or giving or receiving medical care. ${ }^{2}$ Social distancing measures continued to be implemented during this time. Easing of restrictions varied by jurisdiction. ${ }^{3}$

During the lockdown, a national online survey was conducted in Australia, which found that despite significant declines in sexual activity compared to $2019,7.8 \%$ (42/540) of respondents had engaged in sex with casual partners. ${ }^{4}$ Likewise, a sexual health clinic in Melbourne reported a $41 \%$ reduction in consultations with patients presenting with symptoms during the lockdown and a $68 \%$ reduction in the number of consultations
\end{abstract}

\begin{abstract}
Objective: Examine the changes in service delivery Australian public sexual health clinics made to remain open during lockdown.

Methods: A cross-sectional survey designed and delivered on Qualtrics was emailed to 21 directors of public sexual health clinics across Australia from July-August 2020 and asked about a variety of changes to service delivery. Descriptive statistics were calculated.

Results: Twenty clinics participated, all remained open and reported service changes, including suspension of walk-in services in eight clinics. Some clinics stopped offering asymptomatic screening for varying patient populations. Most clinics transitioned to a mix of telehealth and face-to-face consultations. Nineteen clinics reported delays in testing and 13 reported limitations in testing. Most clinics changed to phone consultations for HIV medication refills $(n=15)$ and eleven clinics prescribed longer repeat prescriptions. Fourteen clinics had staff redeployed to assist the COVID-19 response.

Conclusion: Public sexual health clinics pivoted service delivery to reduce risk of COVID-19 transmission in clinical settings, managed staffing reductions and delays in molecular testing, and maintained a focus on urgent and symptomatic STI presentations and those at higher risk of HIV/STI acquisition.

Implications for public health: Further research is warranted to understand what impact reduced asymptomatic screening may have had on community STI transmission.

Key words: service delivery, public health

for asymptomatic screening. ${ }^{5}$ Despite the significant declines, there were still 4,652 consultations during the lockdown at this clinic alone and no significant reduction in sexually transmitted infection (STI) diagnoses

with more concerning symptoms, including infectious syphilis and pelvic inflammatory disease. ${ }^{5}$ Due to the continuation of sexual activity and transmission of STIs, access to sexual health services remained important
\end{abstract}

1. Central Clinical School, Monash University, Melbourne, Victoria

2. Melbourne Sexual Health Centre, Alfred Health, Melbourne, Victoria

3. Kirby Institute, UNSW Sydney, Sydney, New South Wales

4. Sydney Sexual Health Centre, Sydney, New South Wales

5. South Terrace Clinic, Fremantle Hospital and Health Service, Western Australia

6. Department of Sexual Health Medicine, Sydney Local Health District, Camperdown, New South Wales

7. Statewide Sexual Health Services, Tasmania

8. Adelaide Sexual Health Centre, Adelaide, South Australia

9. Sexual Health \& Blood Borne Virus Unit of Centre for Disease Control, Northern Territory Department of Health, Northern Territory

10. Cairns Sexual Health Service, Cairns North, Queensland

11. Queensland Health, Brisbane, Queensland

12. Centre for Population Health, NSW Health, New South Wales

13. Canberra Hospital, Canberra, Australian Capital Territory

14. Melbourne School of Population and Global Health, The University of Melbourne, Victoria

Correspondence to: Dr Tiffany R Phillips, Melbourne Sexual Health Centre, 580 Swanston Street, Carlton, VIC 3053; e-mail: tiffany.phillips@monash.edu

Submitted: March 2021; Revision requested: July 2021; Accepted: August 2021

The authors have stated they have no conflicts of interest.

This is an open access article under the terms of the Creative Commons Attribution-NonCommercial-NoDerivs License, which permits use and distribution in any medium, provided the original work is properly cited, the use is non-commercial and no modifications or adaptations are made. 
during the national lockdown. However, sexual health clinics across Australia had to make service changes in order to continue providing STI and HIV screening and treatment while reducing the risk of COVID-19 transmission among staff and patients. Sexual health service changes across Australia in response to COVID-19 would have been dictated by State Governments, hospital directives and clinical staff decisions depending on the clinic, which makes it difficult to know what changes occurred on a national level.

The aim of this study was to determine the changes in service delivery to patient populations attending for consultations during the nationwide lockdown as well as service changes broadly adopted by Australian sexual health clinics in order to remain open to patients during the lockdown period between March and May 2020. Understanding how sexual health clinics adapted to the COVID-19 lockdown, and which patient populations were affected, will have implications for HIV/STI management.

\section{Methods}

From July to August 2020, 21 sexual health clinic directors who were part of the Australian Collaboration for Coordinated Enhanced Sentinel Surveillance of Sexually Transmissible Infections and Blood-borne Viruses (ACCESS) network ${ }^{6}$ were emailed an invitation to participate in an online survey aimed to document the changes their sexual health service made during the nationwide lockdown. The ACCESS network includes a variety of sites that deliver sexual health services including sexual health clinics and hospitals, general practitioners with high case-loads of patients attending for sexual health and community sexual health centres. For this study we chose to investigate only the sexual health clinics whose primary focus was sexual health service delivery. Sexual health clinics are publicly funded clinics which provide free STI and HIV testing and treatment, and no referral is required. Some clinics may offer services for a specific priority population (e.g. men who have sex with men [MSM]) but generally clinics see all types of patients with a priority given to offering consultations for people with symptoms and in high-risk populations (e.g. MSM, young people, sex workers and Aboriginal or Torres Strait Islanders). ${ }^{7}$ Survey questions were piloted with six sexual health clinics, after which a question on consultations offered for patients attending for vaccinations was split into two questions to distinguish between vaccinations for people living with HIV and people not living with HIV.

The survey was generated, and the data was collected using Qualtrics (Qualtrics, Provo, UT, USA). A downloadable Participant Information Sheet (PIS) was provided on the first page of the survey. Participants needed to agree by clicking 'yes' to a prompt asking if they had read the PIS before the survey questions were shown. The survey asked about a variety of changes made during the lockdown (March-May), including changes in consultations offered to priority patient populations (those with increased risks of STIs), patient intake procedures, hours of operation, and reductions in staff and outreach services. The survey took approximately 15 minutes to complete. Participants were informed that their names and the location and name of their service would not be included in any published material from this study unless they wished to be acknowledged. Descriptive statistics for patient intake changes (e.g. proportion seen by booked appointments vs walk-in) were calculated using Stata (version 13; College Station, TX, USA). This project was approved by Alfred Hospital Ethics Committee, Melbourne, Australia; project number 373/20 and The Human Research and Ethics Committee (HREC) of the Northern Territory Department of Health and Menzies School of Health Research (TEHREC): 2020-3770.

\section{Results}

Twenty of the 21 sexual health services invited to participate completed the survey. All 20 participating clinics remained open for both face-to-face and telehealth consultations during the national lockdown. Most of the clinics were located in New South Wales $(11 / 20 ; 55 \%)$, two were in Victoria (10\%; [2/20]), two were in Queensland (10\%; [2/20]), and one each in the remaining jurisdictions (South Australia, Western Australia, Northern Territory, Australian Capital Territory and Tasmania). There were 12 clinics (60\%; $[12 / 20])$ that serviced only urban areas, five (25\%; [5/20]) regional/remote clinics, and three (15\%; [3/20]) that serviced both urban and regional/remote areas. Below we present findings from clinics with regards to changes in service delivery to a) clinic operations and staff, b) patient intake and consultation procedures, c) changes to consultation types offered to priority populations, d) STI sample collection and testing, and e) COVID-19 screening and prevention measures.

\section{Clinic operations and staff}

The majority of clinics (75\%; [15/20]) reported no reduction to their operating hours during the COVID-19 lockdown, including the five regional/remote services, and two of the services that service both urban and regional/ remote. Five clinics reported reducing their opening hours; two of which stopped offering evening clinics and one stopped offering all evening and Saturday clinics (Table 1). Fifteen clinics offered counselling services at the time of the COVID lockdown, all of which switched to phone/telehealth consultations.

Thirteen clinics (65.0\%; [13/20]) reported specialist and/or outreach clinics associated with their main clinic, of which six reported no change to the specialist and/or outreach clinic during lockdown (Table 1). The remaining clinics with these services saw a shift to telehealth only (5 clinics), or stopped altogether. One clinic indicated that the cessation of outreach/specialist services had a $30 \%$ reduction in service delivery for remote parts of Australia.

Fourteen clinics (70.0\%; [14/20]) indicated a reduction in total number of full-time equivalent (FTE) clinical nurses working in STI services from 74.4 to 45.6 collective FTE, among the clinics who provided an estimation $(n=11)$. There were only three clinics (15.0\%; [3/20]) that had a reduction in total number of full-time equivalent (FTE) clinical doctors during lockdown, from 20.1 to 17.1 FTE collectively (Supplementary Table 1).

Fourteen clinics (70.0\%; [14/20]) had staff redeployed to other sites or redirected to COVID-19 related duties within the clinic during lockdown, most often to public health units (7 clinics), COVID-19 contact tracing (7 clinics), COVID-19 testing clinics (7 clinics), wards for COVID-19 patients (2 clinics) and COVID-19 results phone line (2 clinics).

\section{Patient intake and consultation procedures}

During the lockdown there was an increase in the mean proportion of sexual health clinic patients who were seen by booked appointment as opposed to walk-in, whose sexual history was taken over the phone, and who had a telehealth consultation followed by a face-to-face consultation (Supplementary Table 2). 
Walk-in services for people attending for sexual health care were suspended in eight (40\%; [8/20]) clinics during the lockdown. In the remaining 12 clinics, the proportion of patients seen by walk-in reduced by approximately $19 \%$ (from an overall mean of $50 \%$ of patients to $31 \%$ during the nationwide lockdown). Most clinics (70.0\%; [14/20]) saw an increased proportion of patients by booked appointments during the lockdown. All but one clinic (19 clinics) increased the proportion of patients who had their sexual histories taken over the phone rather than face-to-face (mean $10.8 \%$ vs $56.9 \%$ during lockdown). There were 12 (60\%) clinics that reported having a separate HIV service, and five of those reported only accepting patients by booked appointment during lockdown. Fifteen clinics (75\%; [15/20]) made efforts to reduce face-to-face consultation time, with most of those $(50 \%$; [10/20]) utilising phone consultations followed by face-to-face consultations where necessary.

\section{Changes to consultation types offered to priority populations}

Most clinics reported the number of patients presenting to the clinic for care during the lockdown was 'moderately' or 'greatly' reduced (60\%; [12/20 clinics]) and seven (35\%) said demand was 'somewhat' reduced. Only one clinic reported no reduction in the overall number of patients. All clinics reported a reduction in the number of consultations offered during lockdown, with most clinics reporting moderately or greatly reduced number of consultations (65\%; [13/20]), and 7 (35\%) clinics reporting the number of consultations was only somewhat reduced.

Most clinics described changes in the types of consultations they offered during the lockdown based on patient population and reason for consultation (Table 2). In many clinics, consultations for asymptomatic patients, particularly for heterosexuals, were not offered during the lockdown. When consultations were offered, most clinics adopted a mix of telehealth and face-to-face.

\section{STI sample collection and testing}

Prior to the lockdown, all clinics offered selfcollection for anal swabs for STI screening and most (85\%; [17/20]) offered self-collected vaginal swabs, however there was an increase in the number of clinics who offered selfcollection for oropharyngeal swabs during the lockdown (from 25\%; [5/20] to 85\%; [17/20]) (Table 3).

\begin{tabular}{lcc} 
Table 1: Changes to clinic operations. & & \\
& $\begin{array}{c}\text { Before COVID-19 lockdown } \\
\text { Number of clinics n/N (\%) }\end{array}$ & $\begin{array}{c}\text { During COVID-19 lockdown } \\
\text { Number of clinics n/N (\%) }\end{array}$ \\
\hline Counselling services offered & $15 \mathrm{a} / 19(79 \%)$ & $15 / 19$ (79\%) \\
Switched to telehealth during lockdown & $\mathrm{N} / \mathrm{A}$ & $15 / 15(100 \%)$ \\
Fewer counselling sessions occurred during lockdown & $\mathrm{N} / \mathrm{A}$ & $4 / 15(27 \%)$ \\
\hline Reduced operating hours & $\mathrm{N} / \mathrm{A}$ & $5 / 20(25 \%)$ \\
Evening clinics terminated during lockdown & $\mathrm{N} / \mathrm{A}$ & $3 / 5(60 \%)$ \\
Saturday clinic terminated during lockdown & $\mathrm{N} / \mathrm{A}$ & $1 / 5(20 \%)$ \\
\hline Specialist clinic offered & $9 / 20(45 \%)$ & $1 / 9(11 \%)$ \\
Specialist clinic terminated during lockdown & $\mathrm{N} / \mathrm{A}$ & $5 / 9(56 \%)$ \\
Specialist clinic operation reduced during lockdown & $\mathrm{N} / \mathrm{A}$ & \\
\hline Outreach clinic offered & $12 / 20(60 \%)$ & $3 / 12(25 \%)$ \\
Outreach clinic terminated during lockdown & $\mathrm{N} / \mathrm{A}$ & $4 / 12(33 \%)$ \\
Outreach clinic operation reduced during lockdown & $\mathrm{N} / \mathrm{A}$ & \\
\hline
\end{tabular}
Note:

a: One clinic had a vacant counsellor position prior to lockdown and has thus not been included in the total for clinics offering counselling services.

\begin{tabular}{|c|c|c|c|c|}
\hline Patient population/ presentation & $\begin{array}{c}\text { No or deferred } \\
\text { consultation } \\
\mathrm{n} / \mathrm{N}(\%)\end{array}$ & $\begin{array}{c}\text { Only } \\
\text { Telehealth } \\
\text { consultations } \\
\mathrm{n} / \mathrm{N}^{\mathrm{a}}(\%)\end{array}$ & $\begin{array}{c}\text { Telehealth and } \\
\text { face-to-face } \\
\text { consultations } \\
\mathrm{n} / \mathrm{N}^{\mathrm{a}}(\%)\end{array}$ & $\begin{array}{c}\text { Only } \\
\text { face-to-face } \\
\text { consultations } \\
\mathrm{n} / \mathrm{N}^{\mathrm{a}}(\%)\end{array}$ \\
\hline Anogenital wart treatment & $9 / 19(47)$ & $0 / 10$ & $3 / 10(30)$ & $7 / 10(70 \%)$ \\
\hline Test of re-infection & $7 / 19(37)$ & $1 / 12(8 \%)$ & $9 / 12(75 \%)$ & $2 / 12(17 \%)$ \\
\hline Asymptomatic heterosexual screening & 9/17 (52\%) & $1 / 8(13 \%)$ & $6 / 8(75 \%)$ & $1 / 8(13 \%)$ \\
\hline Asymptomatic MSM screening & $3 / 19(16 \%)$ & $2 / 16(13 \%)$ & $11 / 16(69 \%)$ & $3 / 16(19 \%)$ \\
\hline Asymptomatic transgender screening & $2 / 19(11 \%)$ & $1 / 17(6 \%)$ & $12 / 17(71 \%)$ & $4 / 17(24 \%)$ \\
\hline Asymptomatic PrEP initiation & $0 / 19$ & $0 / 19$ & $14 / 19(74 \%)$ & $5 / 19(26 \%)$ \\
\hline Routine PrEP care & $0 / 19$ & $3 / 19(16 \%)$ & $15 / 19(79 \%)$ & $1 / 19(5 \%)$ \\
\hline Contacts of an STI & $0 / 19$ & $0 / 19$ & $16 / 19(84 \%)$ & $3 / 19(16 \%)$ \\
\hline Person with STI symptoms (e.g. penile or vaginal discharge) & $0 / 19$ & $0 / 19$ & $10 / 19(53 \%)$ & $9 / 19(47 \%)$ \\
\hline Asymptomatic person attending for PEP & $1 / 19(5 \%)$ & $0 / 18$ & $13 / 18(72 \%)$ & $5 / 18(28 \%)$ \\
\hline
\end{tabular}

Note:

One participating clinic did not answer this question ( $N=19)$.

a:Total number of participating clinics is excluding the number of clinics that did not provide consultations for each patient population and presentation (subtracting the first column)

MSM: Men who have sex with men; STI: Sexually transmitted infection; PrEP: HIV Pre-exposure prophylaxis; PEP: HIV post-exposure prophylaxis

All but one clinic reported delays in testing and 13 reported limitations in testing during lockdown; of which seven clinics reported delays only in nucleic acid amplification testing (NAAT) results including Mycoplasma genitalium (but not Chlamydia trachomatis or Neisseria gonorrhoeae). Seven clinics reported reduction in frequency or cessation of laboratory-performed diagnostic assays (including herpes simplex virus, lymphogranuloma venereum, Mycoplasma genitalium, trichomononass vaginalis, and hepatitis $C$ virus genotyping). One clinic's pathology provider suspended $N$. gonorrhoeae cultures during the lockdown due to limitations in human resources.

Eight clinics reported no change to blood tests and eight reported postponing blood tests (e.g. annual bloods instead of standard bi-annual for stable patients living with HIV).
Four clinics described re-directing some patients attending for blood tests to local pathology centres, but no clinic described redirecting all patients for blood tests.

Most clinics changed to phone consultations for HIV medication refill $(n=15)$ with faxed $(n=14)$ or mailed $(n=13)$ prescriptions. Eleven clinics prescribed longer repeat prescriptions (e.g. 12 months' supply instead of six months for stable HIV patients).

\section{COVID-19 screening and prevention measures}

All clinics adopted a variety of COVID-19 screening and prevention measures to minimise transmission of COVID-19 (Table 4).

Staff usage of personal protective equipment (PPE) varied greatly across clinics, as did the situations wherein staff used any PPE, but most commonly were with patients who 
were screened as high-risk of COVID-19 due to contacts or travel, those with COVID-19 symptoms, and those with high risk or symptoms who were having a throat swab. There were two clinics where clinicians did not wear additional PPE during face-to-face consultations and three who adopted PPE for all patients.

\section{Discussion}

This study explored the range of service adaptations sexual health clinics across Australia made during the nationwide lockdown due to COVID-19 in March-May 2020. Our findings show all but one clinic estimated a reduction in number of patients

\section{Table 3: Changes to sample collection, testing and medication delivery.}

Before COVID-19 lockdown During COVID-19 lockdown Number of clinics $n / \mathbb{N}(\%) \quad$ Number of clinics $n / N(\%)$

\begin{tabular}{|c|c|c|}
\hline \multicolumn{3}{|l|}{ Offered ${ }^{\mathrm{a}}$ self-collection for } \\
\hline Anal swabs & $20 / 20(100 \%)$ & $20 / 20(100 \%)$ \\
\hline Vaginal swabs & $17 / 20(85 \%)$ & 18/20 (90\%) \\
\hline Oropharyngeal swabs & $5 / 20(25 \%)$ & $17 / 20(85 \%)$ \\
\hline Reported delays in testing from laboratory & $\mathrm{N} / \mathrm{A}$ & 19/20 (95\%) \\
\hline Reported limitations in testing & N/A & $13 / 20(65 \%)$ \\
\hline \multicolumn{3}{|l|}{ Changes to blood tests } \\
\hline No changes & N/A & $8 / 20(40 \%)$ \\
\hline $\begin{array}{l}\text { Postponed blood tests for stable patients living with HIV (i.e. } \\
\text { annual instead of } 6 \text { months) }\end{array}$ & $N / A$ & $8 / 19^{b}(42 \%)$ \\
\hline Re-directed some patients to local pathology & N/A & $4 / 20(20 \%)$ \\
\hline \multicolumn{3}{|l|}{ Changes to medication delivery } \\
\hline Phone consultation for HIV medication refill & N/A & $15 / 20(75 \%)$ \\
\hline Prescription mailed & N/A & $13 / 20(65 \%)$ \\
\hline Prescription faxed to pharmacy & $\mathrm{N} / \mathrm{A}$ & $14 / 20(70 \%)$ \\
\hline $\begin{array}{l}\text { Prescription scanned /photographed and emailed/SMS to } \\
\text { pharmacy }\end{array}$ & $\mathrm{N} / \mathrm{A}$ & $9 / 20(45 \%)$ \\
\hline Longer prescriptions (e.g. 12 months supply for stable patients) & $N / A$ & $11 / 20(55 \%)$ \\
\hline Medication mailed/other home delivery & $\mathrm{N} / \mathrm{A}$ & 9/20(45\%) \\
\hline No change in medication delivery & N/A & $2 / 20(10 \%)$ \\
\hline
\end{tabular}

Notes:

a: Offered to all who need it

b:One clinic took annual bloods for stable patients living with HIV before COVID-19 lockdown

\begin{tabular}{lc}
\hline Table 4: COVID-19 screening and prevention measures. & \\
\hline Screened patients for COVID-19 & Number of clinics n/N (\%) \\
Temperature check & $20 / 20(100 \%)$ \\
Travel history & $14 / 20(70 \%)$ \\
COVID-19 symptoms & $20 / 20(100 \%)$ \\
Contact of COVID-19 & $20 / 20(100 \%)$ \\
\hline Screening occurred & $18 / 20(90 \%)$ \\
\hline Over the phone/SMS before patient arrived to clinic & \\
At reception & $19 / 20(95 \%)$ \\
At triage & $17 / 20(85 \%)$ \\
During consultations with clinician & $13 / 20(65 \%)$ \\
Entrance to clinic before patient allowed entry & $8 / 20(40 \%)$ \\
\hline Limited number of patients in waiting areas & $3 / 20(15 \%)$ \\
\hline No patients allowed in waiting area & $19 / 20(95 \%)$ \\
\hline Social distancing signs places & $4 / 20(20 \%)$ \\
\hline Chairs removed to facilitate distancing & $18 / 20(90 \%)$ \\
\hline Patients asked to wait outside clinic until telephoned & $17 / 20(85 \%)$ \\
\hline Staff usage of personal protective equipment ${ }^{\mathrm{a}}$ & $13 / 20(65 \%)$ \\
\hline Surgical mask & \\
\hline Scrubs/gown/apron/suit & $16 / 20(80 \%)$ \\
\hline Protective eye wear & $12 / 20(60 \%)$ \\
\hline Not: & $9 / 20(45 \%)$ \\
\hline
\end{tabular}

Note:

a:Staff usage of additional PPE varied between clinics and even within clinics. Some staff used PPE for certain patients (e.g. those at-risk of COVID-19 infection) or for some procedures (e.g. taking throat swabs) presenting for care and a reduction in number of available consultations during the lockdown. Clinics also altered the types of consultations provided in an effort to reduce the number of patients in attendance, predominantly by suspending or deferring consultations to asymptomatic heterosexuals and for anogenital wart treatment. Clinics adopted a variety of measures to reduce the transmission of COVID-19 among staff and patients, from changes in triage protocols and waiting areas to the clinical staff use of PPE, while also managing reductions in FTE clinical staff.

It is unclear what impact many of the service adaptations will have on community transmission of HIV/STIs. The reductions in numbers of patients attending for screening reported in this study could be a result of people having less casual sex in lockdown. While in some states it remained legal to see a non-cohabitating intimate partner during lockdown, ${ }^{8}$ sexual health organisations such as AIDS Council of New South Wales (ACON) and Thorne Harbour Health (VIC) recommended minimising casual sex during COVID-19.9,10 Indeed, a national survey on sex practices during the lockdown among 965 Australian adults found $53.5 \%$ reporting less sex in March-May 2020 compared to 2019, and only $7.8 \%$ engaging in sex with casual partners. ${ }^{11}$ Similar declines in casual sex have been reported among men who have sex with men (MSM), with one Australian study reporting an $84 \%$ reduction in casual sex among MSM during the lockdown ${ }^{12}$ and another study of HIV PrEP users in Victoria showing one-in-four discontinued HIV PrEP during the lockdown. ${ }^{13}$ An Australian national study found that $41.8 \%$ suspended PrEP during the COVID-19 lockdown. ${ }^{14}$

In addition to the reduction in casual sex, a study at a sexual health clinic in Melbourne showed reductions in diagnoses of infections with mild symptoms during lockdown compared with pre-lockdown, such as non-gonococcal urethritis and candidiasis while no changes were seen in presentations of infections with marked symptoms (i.e. pelvic inflammatory disease and symptomatic syphilis), ${ }^{5}$ suggesting sexually active individuals prioritised attending clinic only when they had more intrusive symptoms. Furthermore, there were no changes in the interval between symptom onset and presentation to the clinic, indicating lockdown did not delay healthcare seeking. ${ }^{5}$ Other countries, 
such as America and Italy, have reported similar declines in asymptomatic but not symptomatic STI diagnoses during the COVID-19 pandemic, ${ }^{15-17}$ though variations were seen in Italy, with a sexual health clinic in Rome reporting a reduction in primary syphilis during the first quarter of 2020 during their COVID-19 lockdown. ${ }^{18}$ Further research is needed into the impact the reductions in outreach and specialist clinics may have on priority populations, particularly in rural and remote areas in Australia.

The change to self-collection of oropharyngeal swabs in clinics across Australia is unlikely to impact diagnoses of Chlamydia trachomatis and Neisseria gonorrhoeae given a previous study in MSM showed no difference in positivity after self-collection in either infection. ${ }^{19}$ However, there was an increase in the proportion of indeterminate results for $N$. gonorrhoeae but not $C$. trachomatis during the self-collection period compared to clinician-collected $(1.6 \%$ $[24 / 1,497]$ versus $0.9 \%[23 / 2,600] ; p=0.038),{ }^{19}$ perhaps due to men not collecting sample thoroughly enough which may potentially translate to an increase in extra clinic visits for repeat tests nationwide for oropharyngeal gonorrhoea. Further studies will be required to minimise indeterminate results from selfcollected oropharyngeal swabs.

The increase in the utilisation of telehealth across Australian sexual health services was integral in the response to COVID-19 in terms of reducing consultation times and limiting patient numbers attending clinics for counselling. No studies in Australia, to our knowledge, have investigated the effectiveness of telehealth for sexual health counselling, however, telehealth for mental health services was widely adopted in Australia during the lockdown. ${ }^{20} \mathrm{~A}$ pilot study of telehealth counselling for 14 African American men living with HIV found that telehealth was acceptable for delivering medication counselling and improved participant's health education. ${ }^{21}$ The uptake in telehealth consultations among sexual health services across Australia corresponds with the telehealth growth for general practice consultations. ${ }^{22} \mathrm{~A}$ recent report of Medicare Benefits Schedule (MBS) claims per week from March (when telehealth was included as an item number in the MBS) to September 2020 showed telephone consultations comprised $37.8 \%$ of the weekly total MBS claims in Victoria and $26.9 \%$ in New South Wales. ${ }^{22}$
Further research is required to understand which service adaptations that may be more patient-friendly may be feasibly retained after the COVID-19 pandemic is over, such as continued use of telehealth and increasing intervals for testing for stable patients living with HIV.

There were a couple of limitations to our study. The main limitation was, given the variations in populations and sexual health services available between the Australian states, it was difficult to extrapolate the impact of service adaptations to priority populations seeking sexual health care. However, this study is essential as it provides an overview of the changes made Australiawide and helps direct targeted research in exploring the impact service changes may have had on STI/HIV in Australia. Secondly, our survey was disseminated in August 2020, and hence recall bias might have occurred among the participants.

\section{Implications for public health}

Australian public sexual health clinics rapidly pivoted service delivery to reduce the risk of COVID-19 transmission in their clinical settings, managed staffing reductions and delays in molecular testing, released staff to support the COVID-19 response, such as contact tracing, and maintained a focus on urgent STI presentations and those at higher risk of HIV acquisition. Further research is needed to understand what impact reduced asymptomatic screening may have had on community STI transmission.

\section{Acknowledgements}

We would like to acknowledge all of the participants who completed the survey, including but not limited to: Emanuel Vlahakis, Joanne Lenton, Chris Carmody, David J Smith, and Rochelle Hamilton.

\section{Funding}

E.P.F.C is supported by an Australian National Health and Medical Research Council (NHMRC) Emerging Leadership Investigator Grant (GNT1172873). C.K.F is supported by an Australian NHMRC Leadership Investigator Grants (GNT1172900). JJO is supported by an Australian NHMRC Early Career Fellowship (GNT1104781)

\section{Reference}

1. Prime Minister of Australia. Update On Coronavirus Measures - Media Statement 22 March 2020 [Internet]. Canberra (AUST): Department of the Prime Minister and Cabinet; 2020 [cited 2020 Sep 18]. Available from: https://www.pm.gov.au/media/update-coronavirusmeasures-220320

2. Prime Minister of Australia. Update On Coronavirus Measures - Media Statement 24 March 2020 [Internet]. Canberra (AUST): Department of the Prime Minister and Cabinet; 2020 [cited 2020 Sep 18]. Available from: https://www.pm.gov.au/media/update-coronavirusmeasures-24-March-2020

3. Storen R, Corrigan N. COVID-19: A Chronology of State and Territory Government Announcements (Up Until 30 June 2020). Chronology Research Paper Series, 2020-21. Canberra (AUST): Australian Department of Parliamentary Services; 2020.

4. Coombe J, Kong FYS, Bittleston $\mathrm{H}$, Williams $\mathrm{H}$, Tomnay $J$, Vaisey A, et al. Love during lockdown: Findings from an online survey examining the impact of COVID-19 on the sexual health of people living in Australia. Sex Transm Infect. 2021;97(5):357-62.

5. Chow EPF, Hocking JS, Ong JJ, Phillips TR, Fairey CK. Sexually transmitted infection diagnoses and access to a sexual health service before and after the national lockdown for COVID-19 in Melbourne, Australia. Open Forum Infect Dis. 2020;8(1):ofaa536.

6. Callander D, Moreira C, El-Hayek C, Asselin J, van Gemert C, Watchirs Smith L, et al. Monitoring the control of sexually transmissible infections and blood-borne viruses: Protocol for the Australian Collaboration for Coordinated Enhanced Sentinel Surveillance (ACCESS). JMIR Res Protoc. 2018;7(11):e11028.

7. Ali H, Donovan B, Fairley CK, Chen MY, O'Connor $C C$, Grulich $A E$, et al. Increasing access by priority populations to Australian sexual health clinics. Sex Transm Dis. 2013;40(10):819-21.

8. Eddie R. 'Obvious in retrospect': Rules for Victorian couples who live apart flipped. The Age. 2020;Apr 1:9:34pm.

9. AIDSCouncil of NSW.COVID-19 and Sex. Sydney (AUST): ACON; 2021.

10. Thorne Harbour Health. Sexand COVID-19. Abbotsford (AUST): TH Health; 2021.

11. Coombe J, Kong F, Bittleston H, Williams H, Tomnay J, Vaisey A, et al. Love during lockdown: Findings from an online survey examining the impact of COVID-19 on the sexual practices of people living in Australia. Sex Transm Infect. 2021;97(5):357-62.

12. Hammoud MA, Maher L, Holt M, Degenhardt L, Jin F, Murphy D, et al. Physical distancing due to COVID-19 disrupts sexual behaviors among gay and bisexual men in Australia: Implications for trends in HIV and other sexually transmissible infections. JAcquir/mmune Defic Syndr. 2020;85(3):309-15

13. Chow EPF, Hocking JS, Ong JJ, Schmidt T, Buchanan $A$, Rodriguez E, et al. Changing the use of HIV preexposure prophylaxis among men who have sex with men during the COVID-19 pandemic in Melbourne, Australia. Open Forum Infect Dis. 2020;7(7):ofaa275.

14. Hammoud MA, Grulich A, Holt M, Maher L, Murphy D, Jin F, et al. Substantial decline in use of HIV preexposure prophylaxis following introduction of COVID-19 physical distancing restrictions in Australia: Results from a prospective observational study of gay and bisexual men. J Acquir Immune Defic Syndr. 2021;86(1):22-30.

15. Cusini M, Benardon S, Vidoni G, Brignolo L, Veraldi S, Mandolini PL. Trend of main STIs during COVID-19 pandemic in Milan, Italy. SexTransm Infect. 2021;97(2):99.

16. Crane MA, Popovic A, Stolbach Al, Ghanem KG Reporting of sexually transmitted infections during the COVID-19 pandemic. Sex Transm Infect. 2021;97(2):1012.

17. Sacchelli L, Viviani F, Orioni G, Rucci P, Rosa S, Lanzoni $A$, et al. Sexually transmitted infections during the COVID-19 outbreak: Comparison of patients referring to the service of sexually transmitted diseases during the sanitary emergency with those referring during the common practice. J Eur Acad Dermatol Venereol. 2020;34(10):e553-e6. 


\section{Phillips et al.}

18. Latini A, Magri F, Dona MG, Giuliani M, Cristaudo A, Zaccarelli M. Is COVID-19 affecting the epidemiology of STIs? The experience of syphilis in Rome. Sex Transm Infect. 2021;97(1):78.

19. Chow EPF, Bradshaw CS, Williamson DA, Hall S, Chen MY, Phillips TR, et al. Changing from clinician-collected to self-collected throat swabs for oropharyngeal gonorrhoea and chlamydia screening among men who have sex with men. JClin Microbiol. 2020;58(9):e0121520.

20. Reay RE, Looi JC, Keightley P. Telehealth mental health services during COVID-19: Summary of evidence and clinical practice. Australas Psychiatry. 2020;28(5):514-16.

21. Saberi P, Yuan P, John M, Sheon N, Johnson MO. A pilot study to engage and counsel HIV-positive African American youth via telehealth technology. AIDS Patient Care STDS. 2013;27(9):529-32.

22. Hardie R-A Sezgin G, Dai Z, Georgiou A. The Uptake of GP Telehealth Services During the COVID-19 Pandemic. Sydney (AUST): Macquarie University COVID-19 General Practice Snapshot; 2020:(1). doi.org/10.25949/ C3HE-F430.

\section{Supporting Information}

Additional supporting information may be found in the online version of this article:

Supplementary Table 1: Reductions in full time equivalent (FTE) staff across clinics during 1 COVID-19 lockdown.

Supplementary Table 2: Patient intake and consultation procedure. 


\section{University Library}

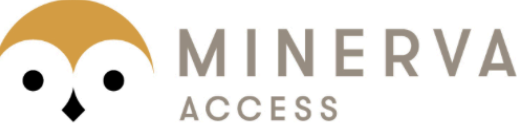

A gateway to Melbourne's research publications

Minerva Access is the Institutional Repository of The University of Melbourne

Author/s:

Phillips, TR;Fairley, CK;Donovan, B;Ong, JJ;McNulty, A;Marshall, L;Templeton, DJ;Owen, L;Ward, A;Gunathilake, M;Russell, D;Langton-Lockton, J;Bourne, C;Martin, S;Chow, EPF

Title:

Sexual health service adaptations to the coronavirus disease 2019 (COVID-19) pandemic in Australia: a nationwide online survey

Date:

2021-09-02

Citation:

Phillips, T. R., Fairley, C. K., Donovan, B., Ong, J. J., McNulty, A., Marshall, L., Templeton, D. J., Owen, L., Ward, A., Gunathilake, M., Russell, D., Langton-Lockton, J., Bourne, C., Martin, S. \& Chow, E. P. F. (2021). Sexual health service adaptations to the coronavirus disease 2019 (COVID-19) pandemic in Australia: a nationwide online survey. AUSTRALIAN AND NEW ZEALAND JOURNAL OF PUBLIC HEALTH, 45 (6), pp.622-627. https:// doi.org/10.1111/1753-6405.13158.

Persistent Link:

http://hdl.handle.net/11343/289630

License:

CC BY-NC-ND 